\title{
Intuitionistic Smooth Bitopological Spaces and Continuity
}

Jin Tae Kim and Seok Jong Lee

Department of Mathematics, Chungbuk National University, Cheongju, Korea

\section{]jfis}

\begin{abstract}
In this paper, we introduce intuitionistic smooth bitopological spaces and the notions of intuitionistic fuzzy semiinterior and semiclosure. Based on these concepts, the characterizations for the intuitionistic fuzzy pairwise semicontinuous mappings are obtained.
\end{abstract}

Keywords: Intuitionistic, Smooth bitopology

\section{Introduction and Preliminaries}

Chang [1] introduced the notion of fuzzy topology. Chang's fuzzy topology is a crisp subfamily of fuzzy sets. However, in his study, Chang did not consider the notion of openness of a fuzzy set, which seems to be a drawback in the process of fuzzification of topological spaces. To overcome this drawback, Šostak [2, 3], based on the idea of degree of openness, introduced a new definition of fuzzy topology as an extension of Chang's fuzzy topology. This generalization of fuzzy topological spaces was later rephrased as smooth topology by Ramadan [4].

Çoker and his colleague [5, 6] introduced intuitionistic fuzzy topological spaces using intuitionistic fuzzy sets which were introduced by Atanassov [7]. Mondal and Samanta [8] introduced the concept of an intuitionistic gradation of openness as a generalization of a smooth topology.

On the other hand, Kandil [9] introduced the concept of fuzzy bitopological spaces as a

Received: Feb. 26, 2014

Revised : Mar. 18, 2014

Accepted: Mar. 19, 2014

Correspondence to: Seok Jong Lee (sjl@cbnu.ac.kr)

(The Korean Institute of Intelligent Systems

cCThis is an Open Access article distributed under the terms of the Creative Commons Attribution Non-Commercial License (http://creativecommons.org/licenses/ by-nc/3.0// which permits unrestricted noncommercial use, distribution, and reproduction in any medium, provided the original work is properly cited. natural generalization of Chang's fuzzy topological spaces. Lee and his colleagues [10, 11] introduced the notion of smooth bitopological spaces as a generalization of smooth topological spaces and Kandil's fuzzy bitopological spaces.

Lim et al. [12] defined the term "intuitionistic smooth topology," which is a slight modification of the intuitionistic gradation of openness of Mondal and Samanta, therefore, it is different from ours.

In this paper, we introduce intuitionistic smooth bitopological spaces and the notions of intuitionistic fuzzy $\left(\mathcal{T}_{i}, \mathcal{T}_{j}\right)-(r, s)$-semiinterior and semiclosure. Based on these concepts, the characterizations for the intuitionistic fuzzy pairwise $(r, s)$-semicontinuous mappings are obtained.

$I$ denotes the unit interval $[0,1]$ of the real line and $I_{0}=(0,1]$. A member $\mu$ of $I^{X}$ is called a fuzzy set in $X$. For any $\mu \in I^{X}, \mu^{c}$ denotes the complement $1-\mu$. By $\tilde{0}$ and $\tilde{1}$ we denote constant mappings on $X$ with value of 0 and 1 , respectively. 
Let $X$ be a nonempty set. An intuitionistic fuzzy set $A$ is an ordered pair

$$
A=\left(\mu_{A}, \gamma_{A}\right)
$$

where the functions $\mu_{A}: X \rightarrow I$ and $\gamma_{A}: X \rightarrow I$ denote the degree of membership and the degree of nonmembership, respectively, and $\mu_{A}+\gamma_{A} \leq 1$. Obviously, every fuzzy set $\mu$ in $X$ is an intuitionistic fuzzy set of the form $(\mu, \tilde{1}-\mu) . I(X)$ denotes a family of all intuitionistic fuzzy sets in $X$ and "IF" stands for intuitionistic fuzzy.

Definition 1.1. ( [4]) A smooth topology on $X$ is a mapping $T: I^{X} \rightarrow I$ which satisfies the following properties:

(1) $T(\tilde{0})=T(\tilde{1})=1$.

(2) $T\left(\mu_{1} \wedge \mu_{2}\right) \geq T\left(\mu_{1}\right) \wedge T\left(\mu_{2}\right)$.

(3) $T\left(\bigvee \mu_{i}\right) \geq \wedge T\left(\mu_{i}\right)$.

The pair $(X, T)$ is called a smooth topological space.

Definition 1.2. ( [11]) A system $\left(X, T_{1}, T_{2}\right)$ consisting of a set $X$ with two smooth topologies $T_{1}$ and $T_{2}$ on $X$ is called a smooth bitopological space.

Definition 1.3. ( [5]) An intuitionistic fuzzy topology on $X$ is a family $T$ of intuitionistic fuzzy sets in $X$ which satisfies the following properties:

(1) $\underline{0}, \underline{1} \in T$.

(2) If $A_{1}, A_{2} \in T$, then $A_{1} \cap A_{2} \in T$.

(3) If $A_{i} \in T$ for each $i$, then $\bigcup A_{i} \in T$.

The pair $(X, T)$ is called an intuitionistic fuzzy topological space.

\section{Intuitionistic Smooth Bitopological Spaces}

Now, we define the notions of intuitionistic smooth topological spaces and intuitionistic smooth bitopological spaces.

Definition 2.1. An intuitionistic smooth topology on $X$ is a mapping $\mathcal{T}: I(X) \rightarrow I$ which satisfies the following properties:

(1) $\mathcal{T}(\underline{0})=\mathcal{T}(\underline{1})=1$.

(2) $\mathcal{T}(A \cap B) \geq \mathcal{T}(A) \wedge \mathcal{T}(B)$.

(3) $\mathcal{T}\left(\bigvee A_{i}\right) \geq \bigwedge \mathcal{T}\left(A_{i}\right)$.

The pair $(X, \mathcal{T})$ is called an intuitionistic smooth topological space.
Let $(X, \mathcal{T})$ be an intuitionistic smooth topological space. For each $r \in I_{0}$, an $r$-cut

$$
\mathcal{T}_{r}=\{A \in I(X) \mid \mathcal{T}(A) \geq r\}
$$

is an intuitionistic fuzzy topology on $X$.

Let $(X, T)$ be an intuitionistic fuzzy topological space and $r \in I_{0}$. Then the mapping $T^{r}: I(X) \rightarrow I$ defined by

$$
T^{r}(A)= \begin{cases}1 & \text { if } \mu=\underline{0}, \underline{1} \\ r & \text { if } A \in T-\{\underline{0}, \underline{1}\} \\ 0 & \text { otherwise }\end{cases}
$$

becomes an intuitionistic smooth topology on $X$.

Definition 2.2. Let $A$ be an intuitionistic fuzzy set in intuitionistic smooth topological space $(X, \mathcal{T})$ and $r \in I_{0}$. Then $A$ is said to be

(1) IF $\mathcal{T}$-r-open if $\mathcal{T}(A) \geq r$,

(2) IF $\mathcal{T}$-r-closed if $\mathcal{T}\left(A^{c}\right) \geq r$.

Definition 2.3. Let $(X, \mathcal{T})$ be an intuitionistic smooth topological space. For $r \in I_{0}$ and for each $A \in I(X)$, the $I F$ $\mathcal{T}$-r-interior is defined by

$$
\mathcal{T} \text {-int }(A, r)=\bigcup\{B \mid B \subseteq A, \mathcal{T}(B) \geq r\}
$$

and the $I F \mathcal{T}$-r-closure is defined by

$$
\mathcal{T}-\operatorname{cl}(A, r)=\bigcap\left\{B \mid A \subseteq B, \mathcal{T}\left(B^{c}\right) \geq r\right\} .
$$

Theorem 2.4. Let $A$ be an intuitionistic fuzzy set in an intuitionistic smooth topological space $(X, \mathcal{T})$ and $r \in I_{0}$. Then

(1) $\mathcal{T}$-int $(A, r)^{c}=\mathcal{T}-\mathrm{cl}\left(A^{c}, r\right)$.

(2) $\mathcal{T}-\operatorname{cl}(A, r)^{c}=\mathcal{T}$-int $\left(A^{c}, r\right)$.

Proof. It follows from Lemma 2.5 in [13].

Definition 2.5. A system $\left(X, \mathcal{T}_{1}, \mathcal{T}_{2}\right)$ consisting of a set $X$ with two intuitionistic smooth topologies $\mathcal{T}_{1}$ and $\mathcal{T}_{2}$ on $X$ is called a intuitionistic smooth bitopological space(ISBTS for short). Throughout this paper the indices $i, j$ take the value in $\{1,2\}$ and $i \neq j$.

Definition 2.6. Let $A$ be an intuitionistic fuzzy set in an ISBTS $\left(X, \mathcal{T}_{1}, \mathcal{T}_{2}\right)$ and $r, s \in I_{0}$. Then $A$ is said to be 
(1) an $\operatorname{IF}\left(\mathcal{T}_{i}, \mathcal{T}_{j}\right)$ - $(r, s)$-semiopen set if there exist an IF $\mathcal{T}_{i^{-}}$ $r$-open set $B$ in $X$ such that $B \subseteq A \subseteq \mathcal{T}_{j}-\operatorname{cl}(B, s)$,

(2) an $I F\left(\mathcal{T}_{i}, \mathcal{T}_{j}\right)-(r, s)$-semiclosed set if there exist an IF $\mathcal{T}_{i}$-r-closed set $B$ in $X$ such that $\mathcal{T}_{j}$-int $(B, s) \subseteq A \subseteq B$.

Theorem 2.7. Let $A$ be an intuitionistic fuzzy set in an ISBTS $\left(X, \mathcal{T}_{1}, \mathcal{T}_{2}\right)$ and $r, s \in I_{0}$. Then the following statements are equivalent:

(1) $A$ is an $\operatorname{IF}\left(\mathcal{T}_{i}, \mathcal{T}_{j}\right)-(r, s)$-semiopen set.

(2) $A^{c}$ is an $\operatorname{IF}\left(\mathcal{T}_{i}, \mathcal{T}_{j}\right)-(r, s)$-semiclosed set.

(3) $\mathcal{T}_{j}-\operatorname{cl}\left(\mathcal{T}_{i}-\operatorname{int}(A, r), s\right) \supseteq A$.

(4) $\mathcal{T}_{j}-\operatorname{int}\left(\mathcal{T}_{i}-\operatorname{cl}\left(A^{c}, r\right), s\right) \subseteq A^{c}$.

Proof. $(1) \Rightarrow(2)$ Let $A$ be an $\left(\mathcal{T}_{i}, \mathcal{T}_{j}\right)-(r, s)$-semiopen set. Then there is an IF $\mathcal{T}_{i}$-r -open set $B$ in $X$ such that $B \subseteq A \subseteq$ $\mathcal{T}_{j}$-cl $(B, s)$. Thus $\mathcal{T}_{j}$-int $\left(B^{c}, s\right) \subseteq A^{c} \subseteq B^{c}$. Since $B^{c}$ is IF $\mathcal{T}_{i}$-r -closed in $X, A^{c}$ is a $\operatorname{IF}\left(\mathcal{T}_{i}, \mathcal{T}_{j}\right)$ - $(r, s)$-semiclosed set in $X$.

(2) $\Rightarrow$ (1) Let $A^{c}$ be an $\operatorname{IF}\left(\mathcal{T}_{i}, \mathcal{T}_{j}\right)$ - $(r, s)$-semiclosed set. Then there is an IF $\mathcal{T}_{i}$-r-closed set $B$ in $X$ such that $\mathcal{T}_{j}$-int $(B, s)$ $\subseteq A^{c} \subseteq B$. Hence $B^{c} \subseteq A \subseteq \mathcal{T}_{j}$-cl $\left(B^{c}, s\right)$. Because $B^{c}$ is IF $\mathcal{T}_{i}$-r-open in $X, A$ is an $\operatorname{IF}\left(\mathcal{T}_{i}, \mathcal{T}_{j}\right)-(r, s)$-semiopen set in $X$.

(1) $\Rightarrow$ (3) Let $A$ be an IF $\left(\mathcal{T}_{i}, \mathcal{T}_{j}\right)-(r, s)$-semiopen set in $X$. Then there exist an IF $\mathcal{T}_{i}$-r -open set $B$ in $X$ such that $B \subseteq A \subseteq \mathcal{T}_{j}-\operatorname{cl}(B, s)$. Since $B$ is IF $\mathcal{T}_{i}$-r -open, we have $B=\mathcal{T}_{i}$-int $(B, r) \subseteq \mathcal{T}_{i}$-int $(A, r)$. Thus

$$
\mathcal{T}_{j}-\operatorname{cl}\left(\mathcal{T}_{i}-\operatorname{int}(A, r), s\right) \supseteq \mathcal{T}_{j}-\operatorname{cl}(B, s) \supseteq A
$$

(3) $\Rightarrow$ (1) Let $\mathcal{T}_{j}-\operatorname{cl}\left(\mathcal{T}_{i}\right.$-int $\left.(A, r), s\right) \supseteq A$ and take $B=$ $\mathcal{T}_{i}$-int $(A, r)$. Then $B$ is an IF $\mathcal{T}_{i}$-r-open set and

$$
\begin{aligned}
B=\mathcal{T}_{i}-\operatorname{int}(A, r) & \subseteq A \\
& \subseteq \mathcal{T}_{j}-\operatorname{cl}\left(\mathcal{T}_{i}-\operatorname{int}(A, r), s\right) \\
& =\mathcal{T}_{j}-\operatorname{cl}(B, s) .
\end{aligned}
$$

Hence $A$ is an $\operatorname{IF}\left(\mathcal{T}_{i}, \mathcal{T}_{j}\right)-(r, s)$-semiopen set.

(3) $\Leftrightarrow$ (4) It follows from Theorem 2.4

Theorem 2.8. Let $A$ be an intuitionistic fuzzy set in an ISBTS $\left(X, \mathcal{T}_{1}, \mathcal{T}_{2}\right)$ and $r, s \in I_{0}$. Then

(1) If $A$ is IF $\mathcal{T}_{1}$-r -open in $\left(X, \mathcal{T}_{1}\right)$, then $A$ is an $\operatorname{IF}\left(\mathcal{T}_{1}, \mathcal{T}_{2}\right)$ $(r, s)$-semiopen set in $\left(X, \mathcal{T}_{1}, \mathcal{T}_{2}\right)$.
(2) If $A$ is IF $\mathcal{T}_{2}$-s-open in $\left(X, \mathcal{T}_{2}\right)$, then $A$ is an $\operatorname{IF}\left(\mathcal{T}_{2}, \mathcal{T}_{1}\right)$ $(s, r)$-semiopen set in $\left(X, \mathcal{T}_{1}, \mathcal{T}_{2}\right)$.

Proof. (1) Let $A$ be an IF $\mathcal{T}_{1}$-r-open set in $\left(X, \mathcal{T}_{1}\right)$. Then $A=\mathcal{T}_{1}$-int $(A, r)$. Thus we have

$$
\mathcal{T}_{2}-\operatorname{cl}\left(\mathcal{T}_{1}-\operatorname{int}(A, r), s\right)=\mathcal{T}_{2}-\operatorname{cl}(A, s) \supseteq A
$$

Hence $A$ is $\operatorname{IF}\left(\mathcal{T}_{1}, \mathcal{T}_{2}\right)$ - $(r, s)$-semiopen in $\left(X, \mathcal{T}_{1}, \mathcal{T}_{2}\right)$.

(2) Similar to (1)

The following example shows that the converses of the above theorem need not be true.

Example 2.9. Let $X=\{x, y\}$ and let $A_{1}, A_{2}, A_{3}$, and $A_{4}$ be intuitionistic fuzzy sets in $X$ defined as

$$
\begin{array}{ll}
A_{1}(x)=(0.1,0.7), & A_{1}(y)=(0.7,0.2) \\
A_{2}(x)=(0.6,0.2), & A_{2}(y)=(0.3,0.6) \\
A_{3}(x)=(0.1,0.7), & A_{3}(y)=(0.9,0.1)
\end{array}
$$

and

$$
A_{4}(x)=(0.7,0.1), A_{4}(y)=(0.3,0.6) .
$$

Define $\mathcal{T}_{1}: I(X) \rightarrow I$ and $\mathcal{T}_{2}: I(X) \rightarrow I$ by

$$
\mathcal{T}_{1}(A)= \begin{cases}1 & \text { if } A=\underline{0}, \underline{1} \\ \frac{1}{2} & \text { if } A=A_{1} \\ 0 & \text { otherwise }\end{cases}
$$

and

$$
\mathcal{T}_{2}(A)= \begin{cases}1 & \text { if } A=\underline{0}, \underline{1} \\ \frac{1}{3} & \text { if } A=A_{2}, \\ 0 & \text { otherwise. }\end{cases}
$$

Then $\left(\mathcal{T}_{1}, \mathcal{T}_{2}\right)$ is an ISBT on $X$. Note that

$$
\mathcal{T}_{2}-\operatorname{cl}\left(\mathcal{T}_{1}-\operatorname{int}\left(A_{3}, \frac{1}{2}\right), \frac{1}{3}\right)=\mathcal{T}_{2}-\operatorname{cl}\left(A_{1}, \frac{1}{3}\right)=\underline{1} \supseteq A_{3}
$$

and

$$
\mathcal{T}_{1}-\operatorname{cl}\left(\mathcal{T}_{2}-\operatorname{int}\left(A_{4}, \frac{1}{3}\right), \frac{1}{2}\right)=\mathcal{T}_{1}-\operatorname{cl}\left(A_{2}, \frac{1}{2}\right)=\underline{1} \supseteq A_{4} .
$$

Hence $A_{3}$ is $\operatorname{IF}\left(\mathcal{T}_{1}, \mathcal{T}_{2}\right)$ - $\left(\frac{1}{2}, \frac{1}{3}\right)$-semiopen and $A_{4}$ is $\operatorname{IF}\left(\mathcal{T}_{2}, \mathcal{T}_{1}\right)$ $\left(\frac{1}{3}, \frac{1}{2}\right)$-semiopen in $\left(X, \mathcal{T}_{1}, \mathcal{T}_{2}\right)$. But $A_{3}$ is not an IF $\mathcal{T}_{1}$ - $\frac{1}{2}$-open set in $\left(X, \mathcal{T}_{1}\right)$ and $A_{4}$ is not an IF $\mathcal{T}_{2}-\frac{1}{3}$-open set in $\left(X, \mathcal{T}_{2}\right)$.

Theorem 2.10. Let $\left(X, \mathcal{T}_{1}, \mathcal{T}_{2}\right)$ be an ISBTS and $r, s \in I_{0}$. Then the following statements are true: 
(1) If $\left\{A_{k}\right\}$ is a family of $\operatorname{IF}\left(\mathcal{T}_{i}, \mathcal{T}_{j}\right)-(r, s)$-semiopen sets in $X$, then $\bigcup A_{k}$ is $\operatorname{IF}\left(\mathcal{T}_{i}, \mathcal{T}_{j}\right)-(r, s)$-semiopen.

(2) If $\left\{A_{k}\right\}$ is a family of $\operatorname{IF}\left(\mathcal{T}_{i}, \mathcal{T}_{j}\right)$ - $(r, s)$-semiclosed sets in $X$, then $\bigcap A_{k}$ is $\operatorname{IF}\left(\mathcal{T}_{i}, \mathcal{T}_{j}\right)-(r, s)$-semiclosed.

Proof. (1) Let $\left\{A_{k}\right\}$ be a collection of $\operatorname{IF}\left(\mathcal{T}_{i}, \mathcal{T}_{j}\right)-(r, s)$-semiopen sets in $X$. Then for each $k$,

$$
A_{k} \subseteq \mathcal{T}_{j}-\mathrm{cl}\left(\mathcal{T}_{i}-\operatorname{int}\left(A_{k}, r\right), s\right)
$$

So we have

$$
\begin{aligned}
\bigcup A_{k} & \subseteq \bigcup \mathcal{T}_{j}-\operatorname{cl}\left(\mathcal{T}_{i}-\operatorname{int}\left(A_{k}, r\right), s\right) \\
& \subseteq \mathcal{T}_{j}-\operatorname{cl}\left(\mathcal{T}_{i}-\operatorname{int}\left(\bigcup A_{k}, r\right), s\right)
\end{aligned}
$$

Thus $\bigcup A_{k}$ is $\operatorname{IF}\left(\mathcal{T}_{i}, \mathcal{T}_{j}\right)-(r, s)$-semiopen.

(2) It follows from (1) using Theorem 2.7.

Definition 2.11. Let $\left(X, \mathcal{T}_{1}, \mathcal{T}_{2}\right)$ be an ISBTS and $r, s \in I_{0}$. For each $A \in I(X)$, the $I F\left(\mathcal{T}_{i}, \mathcal{T}_{j}\right)$ - $(r, s)$-semiinterior is defined by

$$
\begin{aligned}
& \left(\mathcal{T}_{i}, \mathcal{T}_{j}\right)-\operatorname{sint}(A, r, s) \\
& =\bigcup\{B \in I(X) \mid \\
& \left.\quad B \subseteq A, B \text { is } \operatorname{IF}\left(\mathcal{T}_{i}, \mathcal{T}_{j}\right)-(r, s) \text {-semiopen }\right\}
\end{aligned}
$$

and the $I F\left(\mathcal{T}_{i}, \mathcal{T}_{j}\right)-(r, s)$-semiclosure is defined by

$$
\begin{aligned}
& \left(\mathcal{T}_{i}, \mathcal{T}_{j}\right)-\operatorname{scl}(A, r, s) \\
& =\bigcap\{B \in I(X) \mid \\
& \left.A \subseteq B, B \text { is } \operatorname{IF}\left(\mathcal{T}_{i}, \mathcal{T}_{j}\right)-(r, s) \text {-semiclosed }\right\} .
\end{aligned}
$$

Obviously, $\left(\mathcal{T}_{i}, \mathcal{T}_{j}\right)-\operatorname{scl}(A, r, s)$ is the smallest $\operatorname{IF}\left(\mathcal{T}_{i}, \mathcal{T}_{j}\right)$ $(r, s)$-semiclosed set which contains $A$ and $\left(\mathcal{T}_{i}, \mathcal{T}_{j}\right)$-sint $(A, r, s)$ is the greatest $\operatorname{IF}\left(\mathcal{T}_{i}, \mathcal{T}_{j}\right)-(r, s)$-semiopen set which is contained in $A$. Also, $\left(\mathcal{T}_{i}, \mathcal{T}_{j}\right)-\operatorname{scl}(A, r, s)=A$ for any $\operatorname{IF}\left(\mathcal{T}_{i}, \mathcal{T}_{j}\right)-(r, s)$ semiclosed set $A$ and $\left(\mathcal{T}_{i}, \mathcal{T}_{j}\right)$-sint $(A, r, s)=A$ for any IF $\left(\mathcal{T}_{i}, \mathcal{T}_{j}\right)-(r, s)$-semiopen set $A$.

Moreover, we have

$$
\begin{aligned}
\mathcal{T}_{i}-\operatorname{int}(A, r) & \subseteq\left(\mathcal{T}_{i}, \mathcal{T}_{j}\right)-\operatorname{sint}(A, r, s) \\
& \subseteq A \\
& \subseteq\left(\mathcal{T}_{i}, \mathcal{T}_{j}\right)-\operatorname{scl}(A, r, s) \\
& \subseteq \mathcal{T}_{i}-\operatorname{cl}(A, r) .
\end{aligned}
$$

Also, we have the following results:

(1) $\left(\mathcal{T}_{i}, \mathcal{T}_{j}\right)-\operatorname{scl}(\underline{0}, r, s)=\underline{0},\left(\mathcal{T}_{i}, \mathcal{T}_{j}\right)-\operatorname{scl}(\underline{1}, r, s)=\underline{1}$.

(2) $\left(\mathcal{T}_{i}, \mathcal{T}_{j}\right)-\operatorname{scl}(A, r, s) \supseteq A$.

(3) $\left(\mathcal{T}_{i}, \mathcal{T}_{j}\right)-\operatorname{scl}(A, r, s) \cup\left(\mathcal{T}_{i}, \mathcal{T}_{j}\right)-\operatorname{scl}(B, r, s)$ $\subseteq\left(\mathcal{T}_{i}, \mathcal{T}_{j}\right)-\operatorname{scl}(A \cup B, r, s)$.

(4) $\left(\mathcal{T}_{i}, \mathcal{T}_{j}\right)-\operatorname{scl}\left(\left(\mathcal{T}_{i}, \mathcal{T}_{j}\right)-\operatorname{scl}(A, r, s), r, s\right)$ $=\left(\mathcal{T}_{i}, \mathcal{T}_{j}\right)-\operatorname{scl}(A, r, s)$.

(5) $\left(\mathcal{T}_{i}, \mathcal{T}_{j}\right)-\operatorname{sint}(\underline{0}, r, s)=\underline{0},\left(\mathcal{T}_{i}, \mathcal{T}_{j}\right)-\operatorname{sint}(\underline{1}, r, s)=\underline{1}$.

(6) $\left(\mathcal{T}_{i}, \mathcal{T}_{j}\right)-\operatorname{sint}(A, r, s) \subseteq A$.

(7) $\left(\mathcal{T}_{i}, \mathcal{T}_{j}\right)-\operatorname{sint}(A, r, s) \cap\left(\mathcal{T}_{i}, \mathcal{T}_{j}\right)-\operatorname{sint}(B, r, s)$ $\supseteq\left(\mathcal{T}_{i}, \mathcal{T}_{j}\right)-\operatorname{sint}(A \cap B, r, s)$.

(8) $\left(\mathcal{T}_{i}, \mathcal{T}_{j}\right)-\operatorname{sint}\left(\left(\mathcal{T}_{i}, \mathcal{T}_{j}\right)-\operatorname{sint}(A, r, s), r, s\right)$ $=\left(\mathcal{T}_{i}, \mathcal{T}_{j}\right)-\operatorname{sint}(A, r, s)$.

Theorem 2.12. Let $A$ be an intuitionistic fuzzy set in an ISBTS $\left(X, \mathcal{T}_{1}, \mathcal{T}_{2}\right)$ and $r, s \in I_{0}$. Then we have

(1) $\left(\mathcal{T}_{i}, \mathcal{T}_{j}\right)-\operatorname{sint}(A, r, s)^{c}=\left(\mathcal{T}_{i}, \mathcal{T}_{j}\right)-\operatorname{scl}\left(A^{c}, r, s\right)$.

(2) $\left(\mathcal{T}_{i}, \mathcal{T}_{j}\right)-\operatorname{scl}(A, r, s)^{c}=\left(\mathcal{T}_{i}, \mathcal{T}_{j}\right)-\operatorname{sint}\left(A^{c}, r, s\right)$.

Proof. (1) Since

$$
\left(\mathcal{T}_{i}, \mathcal{T}_{j}\right)-\operatorname{sint}(A, r, s) \subseteq A \text { and }\left(\mathcal{T}_{i}, \mathcal{T}_{j}\right)-\operatorname{sint}(A, r, s)
$$

is $\operatorname{IF}\left(\mathcal{T}_{i}, \mathcal{T}_{j}\right)-(r, s)$-semiopen in $X, A^{c} \subseteq\left(\mathcal{T}_{i}, \mathcal{T}_{j}\right)-\operatorname{sint}(A, r, s)^{c}$ and $\left(\mathcal{T}_{i}, \mathcal{T}_{j}\right)-\operatorname{sint}(A, r, s)^{c}$ is $\operatorname{IF}\left(\mathcal{T}_{i}, \mathcal{T}_{j}\right)-(r, s)$-semiclosed. Thus

$$
\begin{aligned}
& \left(\mathcal{T}_{i}, \mathcal{T}_{j}\right)-\operatorname{scl}\left(A^{c}, r, s\right) \\
& \quad \subseteq\left(\mathcal{T}_{i}, \mathcal{T}_{j}\right)-\operatorname{scl}\left(\left(\mathcal{T}_{i}, \mathcal{T}_{j}\right)-\operatorname{sint}(A, r, s)^{c}, r, s\right) \\
& \quad=\left(\mathcal{T}_{i}, \mathcal{T}_{j}\right)-\operatorname{sint}(A, r, s)^{c} .
\end{aligned}
$$

From that $A^{c} \subseteq\left(\mathcal{T}_{i}, \mathcal{T}_{j}\right)-\operatorname{scl}\left(A^{c}, r, s\right)$ and $\left(\mathcal{T}_{i}, \mathcal{T}_{j}\right)-\operatorname{scl}\left(A^{c}, r, s\right)$ is IF $\left(\mathcal{T}_{i}, \mathcal{T}_{j}\right)-(r, s)$-semiclosed, $\left(\mathcal{T}_{i}, \mathcal{T}_{j}\right)-\operatorname{scl}\left(A^{c}, r, s\right)^{c} \subseteq A$ and $\left(\mathcal{T}_{i}, \mathcal{T}_{j}\right)-\operatorname{scl}\left(A^{c}, r, s\right)^{c}$ is $\operatorname{IF}\left(\mathcal{T}_{i}, \mathcal{T}_{j}\right)-(r, s)$-semiopen. Thus we have

$$
\begin{aligned}
& \left(\mathcal{T}_{i}, \mathcal{T}_{j}\right)-\operatorname{scl}\left(A^{c}, r, s\right)^{c} \\
& \quad=\left(\mathcal{T}_{i}, \mathcal{T}_{j}\right)-\operatorname{sint}\left(\left(\mathcal{T}_{i}, \mathcal{T}_{j}\right)-\operatorname{scl}\left(A^{c}, r, s\right)^{c}, r, s\right) \\
& \quad \subseteq\left(\mathcal{T}_{i}, \mathcal{T}_{j}\right)-\operatorname{sint}(A, r, s) .
\end{aligned}
$$

Hence

$\left(\mathcal{T}_{i}, \mathcal{T}_{j}\right)-\operatorname{sint}(A, r, s)^{c} \subseteq\left(\mathcal{T}_{i}, \mathcal{T}_{j}\right)-\operatorname{scl}\left(A^{c}, r, s\right)$. 
Therefore

$$
\left(\mathcal{T}_{i}, \mathcal{T}_{j}\right)-\operatorname{sint}(A, r, s)^{c}=\left(\mathcal{T}_{i}, \mathcal{T}_{j}\right)-\operatorname{scl}\left(A^{c}, r, s\right)
$$

(2) Similar to (1).

\section{Continuity in Intuitionistic Smooth Bitopol- ogy}

We define the notions of IF pairwise $(r, s)$-semicontinuous mappings in intuitionistic smooth bitopological spaces, and investigate their characteristic properties.

Definition 3.1. Let $f:(X, \mathcal{T}) \rightarrow(Y, \mathcal{U})$ be a mapping from an intuitionistic smooth topological spaces $X$ to an intuitionistic smooth topological spaces $Y$ and $r \in I_{0}$. Then $f$ is called an IF r-continuous mapping if $f^{-1}(B)$ is IF $\mathcal{T}$ - $r$-open in $X$ for each IF $\mathcal{U}$-r-open set $B$ in $Y$.

Definition 3.2. Let $f:\left(X, \mathcal{T}_{1}, \mathcal{T}_{2}\right) \rightarrow\left(Y, \mathcal{U}_{1}, \mathcal{U}_{2}\right)$ be a mapping from an ISBTS $X$ to an ISBTS $Y$ and $r, s \in I_{0}$. Then $f$ is said to be IF pairwise $(r, s)$-continuous if the induced mapping $f:\left(X, \mathcal{T}_{1}\right) \rightarrow\left(Y, \mathcal{U}_{1}\right)$ is an IF $r$-continuous mapping and the induced mapping $f:\left(X, \mathcal{T}_{2}\right) \rightarrow\left(Y, \mathcal{U}_{2}\right)$ is an IF $s$-continuous mapping.

Definition 3.3. Let $f:\left(X, \mathcal{T}_{1}, \mathcal{T}_{2}\right) \rightarrow\left(Y, \mathcal{U}_{1}, \mathcal{U}_{2}\right)$ be a mapping from an ISBTS $X$ to an ISBTS $Y$ and $r, s \in I_{0}$. Then $f$ is said to be IF pairwise $(r, s)$-semicontinuous if $f^{-1}(A)$ is an IF $\left(\mathcal{T}_{1}, \mathcal{T}_{2}\right)-(r, s)$-semiopen set in $X$ for each IF $\mathcal{U}_{1}$ - $r$-open set $A$ in $Y$ and $f^{-1}(B)$ is an $\operatorname{IF}\left(\mathcal{T}_{2}, \mathcal{T}_{1}\right)-(s, r)$-semiopen set in $X$ for each IF $\mathcal{U}_{2}$-s-open set $B$ in $Y$.

Remark 3.4. It is obvious that every IF pairwise $(r, s)$-continuou mapping is IF pairwise $(r, s)$-semicontinuous. But the following example shows that the converse need not be true.

Example 3.5. Let $\left(X, \mathcal{T}_{1}, \mathcal{T}_{2}\right)$ be an ISBTS as described in Example 2.9 Define $\mathcal{U}_{1}: I(X) \rightarrow I$ and $\mathcal{U}_{2}: I(X) \rightarrow I$ by

$$
\mathcal{U}_{1}(A)= \begin{cases}1 & \text { if } A=\underline{0}, \underline{1} \\ 0 & \text { otherwise }\end{cases}
$$

and

$$
\mathcal{U}_{2}(A)= \begin{cases}1 & \text { if } A=\underline{0}, \underline{1} \\ \frac{1}{3} & \text { if } A=A_{4} \\ 0 & \text { otherwise }\end{cases}
$$

Then $\left(\mathcal{U}_{1}, \mathcal{U}_{2}\right)$ is an ISBT on $X$. Consider a mapping $f$ : $\left(X, \mathcal{T}_{1}, \mathcal{T}_{2}\right) \rightarrow\left(X, \mathcal{U}_{1}, \mathcal{U}_{2}\right)$ defined by $f(x)=x$ and $f(y)=y$.
Then $f$ is IF pairwise $\left(\frac{1}{2}, \frac{1}{3}\right)$-semicontinuous. But $f$ is not an IF pairwise $\left(\frac{1}{2}, \frac{1}{3}\right)$-continuous mapping.

Theorem 3.6. Let $f:\left(X, \mathcal{T}_{1}, \mathcal{T}_{2}\right) \rightarrow\left(Y, \mathcal{U}_{1}, \mathcal{U}_{2}\right)$ be a mapping from an ISBTS $X$ to an ISBTS $Y$ and $r, s \in I_{0}$. Then the following statements are equivalent:

(1) $f$ is IF pairwise $(r, s)$-semicontinuous.

(2) $f^{-1}(A)$ is an IF $\left(\mathcal{T}_{1}, \mathcal{T}_{2}\right)-(r, s)$-semiclosed set in $X$ for each IF $\mathcal{U}_{1}-r$-closed set $A$ in $Y$ and $f^{-1}(B)$ is an IF $\left(\mathcal{T}_{2}, \mathcal{T}_{1}\right)-(s, r)$-semiclosed set in $X$ for each IF $\mathcal{U}_{2}-s$ closed set $B$ in $Y$.

(3) For each intuitionistic fuzzy set $B$ in $Y$,

$$
\mathcal{T}_{2}-\operatorname{int}\left(\mathcal{T}_{1}-\operatorname{cl}\left(f^{-1}(B), r\right), s\right) \subseteq f^{-1}\left(\mathcal{U}_{1}-\operatorname{cl}(B, r)\right)
$$

and

$$
\mathcal{T}_{1}-\operatorname{int}\left(\mathcal{T}_{2}-\operatorname{cl}\left(f^{-1}(B), s\right), r\right) \subseteq f^{-1}\left(\mathcal{U}_{2}-\operatorname{cl}(B, s)\right)
$$

(4) For each intuitionistic fuzzy set $A$ in $X$,

$$
f\left(\mathcal{T}_{2}-\operatorname{int}\left(\mathcal{T}_{1}-\operatorname{cl}(A, r), s\right)\right) \subseteq \mathcal{U}_{1}-\operatorname{cl}(f(A), r)
$$

and

$$
f\left(\mathcal{T}_{1}-\operatorname{int}\left(\mathcal{T}_{2}-\operatorname{cl}(A, s), r\right)\right) \subseteq \mathcal{U}_{2}-\operatorname{cl}(f(A), s) .
$$

Proof. (1) $\Leftrightarrow$ (2) Trivial.

(2) $\Rightarrow$ (3) Let $B$ be an intuitionistic fuzzy set in $Y$. Then $\mathcal{U}_{1}-\mathrm{cl}(B, r)$ is IF $\mathcal{U}_{1}$-r-closed and $\mathcal{U}_{2}-\operatorname{cl}(B, s)$ is IF $\mathcal{U}_{2}$-s-closed in $Y$. Hence by $(2), f^{-1}\left(\mathcal{U}_{1}-\mathrm{cl}(B, r)\right)$ is an $\operatorname{IF}\left(\mathcal{T}_{1}, \mathcal{T}_{2}\right)-(r, s)$ semiclosed set and $f^{-1}\left(\mathcal{U}_{2}-\operatorname{cl}(B, s)\right)$ is an $\operatorname{IF}\left(\mathcal{T}_{2}, \mathcal{T}_{1}\right)-(s, r)$ semiclosed set in $X$. Thus we obtain

$$
\begin{aligned}
& \mathcal{T}_{2}-\operatorname{int}\left(\mathcal{T}_{1}-\operatorname{cl}\left(f^{-1}(B), r\right), s\right) \\
& \subseteq \mathcal{T}_{2}-\operatorname{int}\left(\mathcal{T}_{1}-\operatorname{cl}\left(f^{-1}\left(\mathcal{U}_{1}-\operatorname{cl}(B, r)\right), r\right), s\right) \\
& \subseteq f^{-1}\left(\mathcal{U}_{1}-\operatorname{cl}(B, r)\right)
\end{aligned}
$$

and

$$
\begin{aligned}
& \mathcal{T}_{1}-\operatorname{int}\left(\mathcal{T}_{2}-\operatorname{cl}\left(f^{-1}(B), s\right), r\right) \\
& \subseteq \mathcal{T}_{1}-\operatorname{int}\left(\mathcal{T}_{2}-\operatorname{cl}\left(f^{-1}\left(\mathcal{U}_{2}-\operatorname{cl}(B, s)\right), s\right), r\right) \\
& \subseteq f^{-1}\left(\mathcal{U}_{2}-\operatorname{cl}(B, s)\right) .
\end{aligned}
$$

(3) $\Rightarrow$ (4) Let $A$ be an intuitionistic fuzzy set in $X$. Then by (3), we have

$$
\begin{aligned}
\mathcal{T}_{2}-\operatorname{int}\left(\mathcal{T}_{1}-\operatorname{cl}(A, r), s\right) & \subseteq \mathcal{T}_{2}-\operatorname{int}\left(\mathcal{T}_{1}-\operatorname{cl}\left(f^{-1}(f(A)), r\right), s\right) \\
& \subseteq f^{-1}\left(\mathcal{U}_{1}-\operatorname{cl}(f(A), r)\right)
\end{aligned}
$$


and

$$
\begin{aligned}
\mathcal{T}_{1}-\operatorname{int}\left(\mathcal{T}_{2}-\operatorname{cl}(A, s), r\right) & \subseteq \mathcal{T}_{1}-\operatorname{int}\left(\mathcal{T}_{2}-\operatorname{cl}\left(f^{-1}(f(A)), s\right), r\right) \\
& \subseteq f^{-1}\left(\mathcal{U}_{2}-\operatorname{cl}(f(A), s)\right) .
\end{aligned}
$$

Hence

$$
f\left(\mathcal{T}_{2}-\operatorname{int}\left(\mathcal{T}_{1}-\operatorname{cl}(A, r), s\right)\right) \subseteq \mathcal{U}_{1}-\operatorname{cl}(f(A), r)
$$

and

$$
f\left(\mathcal{T}_{1}-\operatorname{int}\left(\mathcal{T}_{2}-\operatorname{cl}(A, s), r\right)\right) \subseteq \mathcal{U}_{2}-\operatorname{cl}(f(A), s) .
$$

(4) $\Rightarrow(2)$ Let $A$ be any IF $\mathcal{U}_{1}-r$-closed set and $B$ any IF $\mathcal{U}_{2}$ -s-closed set in $Y$. By (4), we obtain

$$
\begin{aligned}
f\left(\mathcal{T}_{2}-\operatorname{int}\left(\mathcal{T}_{1}-\operatorname{cl}\left(f^{-1}(A), r\right), s\right)\right) & \subseteq \mathcal{U}_{1}-\operatorname{cl}\left(f\left(f^{-1}(A)\right), r\right) \\
& \subseteq \mathcal{U}_{1}-\operatorname{cl}(A, r)=A
\end{aligned}
$$

and

$$
\begin{aligned}
f\left(\mathcal{T}_{1}-\operatorname{int}\left(\mathcal{T}_{2}-\operatorname{cl}\left(f^{-1}(B), s\right), r\right)\right) & \subseteq \mathcal{U}_{2}-\operatorname{cl}\left(f\left(f^{-1}(B)\right), s\right) \\
& \subseteq \mathcal{U}_{2}-\operatorname{cl}(B, s)=B .
\end{aligned}
$$

Hence

$$
\mathcal{T}_{2}-\operatorname{int}\left(\mathcal{T}_{1}-\operatorname{cl}\left(f^{-1}(A), r\right), s\right) \subseteq f^{-1}(A)
$$

and

$$
\mathcal{T}_{1}-\operatorname{int}\left(\mathcal{T}_{2}-\operatorname{cl}\left(f^{-1}(B), s\right), r\right) \subseteq f^{-1}(B) .
$$

Therefore $f^{-1}(A)$ is an $\operatorname{IF}\left(\mathcal{T}_{1}, \mathcal{T}_{2}\right)-(r, s)$-semiclosed set and $f^{-1}(B)$ is an $\operatorname{IF}\left(\mathcal{T}_{2}, \mathcal{T}_{1}\right)-(s, r)$-semiclosed set in $X$.

Theorem 3.7. Let $f:\left(X, \mathcal{T}_{1}, \mathcal{T}_{2}\right) \rightarrow\left(Y, \mathcal{U}_{1}, \mathcal{U}_{2}\right)$ be a mapping from an ISBTS $X$ to an ISBTS $Y$ and $r, s \in I_{0}$. Then the following statements are equivalent:

(1) $f$ is IF pairwise $(r, s)$-semicontinuous.

(2) For each intuitionistic fuzzy set $A$ in $X$,

$$
f\left(\left(\mathcal{T}_{1}, \mathcal{T}_{2}\right)-\operatorname{scl}(A, r, s)\right) \subseteq \mathcal{U}_{1}-\operatorname{cl}(f(A), r)
$$

and

$$
f\left(\left(\mathcal{T}_{2}, \mathcal{T}_{1}\right)-\operatorname{scl}(A, s, r)\right) \subseteq \mathcal{U}_{2}-\operatorname{cl}(f(A), s) .
$$

(3) For each intuitionistic fuzzy set $B$ in $Y$,

$$
\left(\mathcal{T}_{1}, \mathcal{T}_{2}\right)-\operatorname{scl}\left(f^{-1}(B), r, s\right) \subseteq f^{-1}\left(\mathcal{U}_{1}-\operatorname{cl}(B, r)\right)
$$

and

$$
\left(\mathcal{T}_{2}, \mathcal{T}_{1}\right)-\operatorname{scl}\left(f^{-1}(B), s, r\right) \subseteq f^{-1}\left(\mathcal{U}_{2}-\operatorname{cl}(B, s)\right)
$$

(4) For each intuitionistic fuzzy set $B$ in $Y$,

$$
f^{-1}\left(\mathcal{U}_{1}-\operatorname{int}(B, r)\right) \subseteq\left(\mathcal{T}_{1}, \mathcal{T}_{2}\right)-\operatorname{sint}\left(f^{-1}(B), r, s\right)
$$

and

$$
f^{-1}\left(\mathcal{U}_{2}-\operatorname{int}(B, s)\right) \subseteq\left(\mathcal{T}_{2}, \mathcal{T}_{1}\right)-\operatorname{sint}\left(f^{-1}(B), s, r\right)
$$

Proof. (1) $\Rightarrow$ (2) Let $A$ be an intuitionistic fuzzy set in $X$. Then $\mathcal{U}_{1}-\operatorname{cl}(f(A), r)$ is IF $\mathcal{U}_{1}-r$-closed and $\mathcal{U}_{2}-\operatorname{cl}(f(A), s)$ is IF $\mathcal{U}_{2}$-s-closed in $Y$. Since $f$ is IF pairwise $(r, s)$-semicontinuous, $f^{-1}\left(\mathcal{U}_{1}-\operatorname{cl}(f(A), r)\right)$ is an $\operatorname{IF}\left(\mathcal{T}_{1}, \mathcal{T}_{2}\right)-(r, s)$-semiclosed set and $f^{-1}\left(\mathcal{U}_{2}-\mathrm{cl}(f(A), s)\right)$ is an $\operatorname{IF}\left(\mathcal{T}_{2}, \mathcal{T}_{1}\right)-(s, r)$-semiclosed set in $X$. Hence

$$
\begin{aligned}
& \left(\mathcal{T}_{1}, \mathcal{T}_{2}\right)-\operatorname{scl}(A, r, s) \\
& \subseteq\left(\mathcal{T}_{1}, \mathcal{T}_{2}\right)-\operatorname{scl}\left(f^{-1}\left(\mathcal{U}_{1}-\operatorname{cl}(f(A), r)\right), r, s\right) \\
& =f^{-1}\left(\mathcal{U}_{1}-\operatorname{cl}(f(A), r)\right)
\end{aligned}
$$

and

$$
\begin{aligned}
& \left(\mathcal{T}_{2}, \mathcal{T}_{1}\right)-\operatorname{scl}(A, s, r) \\
& \subseteq\left(\mathcal{T}_{2}, \mathcal{T}_{1}\right)-\operatorname{scl}\left(f^{-1}\left(\mathcal{U}_{2}-\operatorname{cl}(f(A), s)\right), s, r\right) \\
& =f^{-1}\left(\mathcal{U}_{2}-\operatorname{cl}(f(A), s)\right) .
\end{aligned}
$$

Therefore

$$
f\left(\left(\mathcal{T}_{1}, \mathcal{T}_{2}\right)-\operatorname{scl}(A, r, s)\right) \subseteq \mathcal{U}_{1}-\operatorname{cl}(f(A), r)
$$

and

$$
f\left(\left(\mathcal{T}_{2}, \mathcal{T}_{1}\right)-\operatorname{scl}(A, s, r)\right) \subseteq \mathcal{U}_{2}-\operatorname{cl}(f(A), s) .
$$

(2) $\Rightarrow(3)$ Let $B$ be an intuitionistic fuzzy set in $Y$. Then by (2), we obtain

$$
\begin{aligned}
f\left(\left(\mathcal{T}_{1}, \mathcal{T}_{2}\right)-\operatorname{scl}\left(f^{-1}(B), r, s\right)\right) & \subseteq \mathcal{U}_{1}-\operatorname{cl}\left(f\left(f^{-1}(B)\right), r\right) \\
& \subseteq \mathcal{U}_{1}-\operatorname{cl}(B, r)
\end{aligned}
$$

and

$$
\begin{aligned}
f\left(\left(\mathcal{T}_{2}, \mathcal{T}_{1}\right)-\operatorname{scl}\left(f^{-1}(B), s, r\right)\right) & \subseteq \mathcal{U}_{2}-\operatorname{cl}\left(f\left(f^{-1}(B)\right), s\right) \\
& \subseteq \mathcal{U}_{2}-\operatorname{cl}(B, s) .
\end{aligned}
$$

Hence

$$
\left(\mathcal{T}_{1}, \mathcal{T}_{2}\right)-\operatorname{scl}\left(f^{-1}(B), r, s\right) \subseteq f^{-1}\left(\mathcal{U}_{1}-\operatorname{cl}(B, r)\right)
$$


and

$$
\left(\mathcal{T}_{2}, \mathcal{T}_{1}\right)-\operatorname{scl}\left(f^{-1}(B), s, r\right) \subseteq f^{-1}\left(\mathcal{U}_{2}-\operatorname{cl}(B, s)\right)
$$

(3) $\Rightarrow$ (4) Let $B$ be an intuitionistic fuzzy set in $Y$. Then by (3), we have

$$
\left(\mathcal{T}_{1}, \mathcal{T}_{2}\right)-\operatorname{scl}\left(f^{-1}\left(B^{c}\right), r, s\right) \subseteq f^{-1}\left(\mathcal{U}_{1}-\operatorname{cl}\left(B^{c}, r\right)\right)
$$

and

$$
\left(\mathcal{T}_{2}, \mathcal{T}_{1}\right)-\operatorname{scl}\left(f^{-1}\left(B^{c}\right), s, r\right) \subseteq f^{-1}\left(\mathcal{U}_{2}-\operatorname{cl}\left(B^{c}, s\right)\right)
$$

Hence

$$
\begin{aligned}
f^{-1}\left(\mathcal{U}_{1}-\operatorname{int}(B, r)\right) & =\left(f^{-1}\left(\mathcal{U}_{1}-\operatorname{cl}\left(B^{c}, r\right)\right)\right)^{c} \\
& \subseteq\left(\mathcal{T}_{1}, \mathcal{T}_{2}\right)-\operatorname{scl}\left(f^{-1}\left(B^{c}\right), r, s\right)^{c} \\
& =\left(\mathcal{T}_{1}, \mathcal{T}_{2}\right)-\operatorname{sint}\left(f^{-1}(B), r, s\right)
\end{aligned}
$$

and

$$
\begin{aligned}
f^{-1}\left(\mathcal{U}_{2}-\operatorname{int}(B, s)\right) & =\left(f^{-1}\left(\mathcal{U}_{2}-\operatorname{cl}\left(B^{c}, s\right)\right)\right)^{c} \\
& \subseteq\left(\mathcal{T}_{2}, \mathcal{T}_{1}\right)-\operatorname{scl}\left(f^{-1}\left(B^{c}\right), s, r\right)^{c} \\
& =\left(\mathcal{T}_{2}, \mathcal{T}_{1}\right)-\operatorname{sint}\left(f^{-1}(B), s, r\right) .
\end{aligned}
$$

(4) $\Rightarrow(1)$ Let $A$ be any IF $\mathcal{U}_{1}-r$-open set and $B$ any IF $\mathcal{U}_{2}-s$ open set in $Y$. Then $\mathcal{U}_{1}$-int $(A, r)=A$ and $\mathcal{U}_{2}-\operatorname{int}(B, s)=B$. Hence

$$
\begin{aligned}
f^{-1}(A) & =f^{-1}\left(\mathcal{U}_{1}-\operatorname{int}(A, r)\right) \\
& \subseteq\left(\mathcal{T}_{1}, \mathcal{T}_{2}\right)-\operatorname{sint}\left(f^{-1}(A), r, s\right) \\
& \subseteq f^{-1}(A)
\end{aligned}
$$

and

$$
\begin{aligned}
f^{-1}(B) & =f^{-1}\left(\mathcal{U}_{2}-\operatorname{int}(B, s)\right) \\
& \subseteq\left(\mathcal{T}_{2}, \mathcal{T}_{1}\right)-\operatorname{sint}\left(f^{-1}(B), s, r\right) \\
& \subseteq f^{-1}(B) .
\end{aligned}
$$

Thus

$$
f^{-1}(A)=\left(\mathcal{T}_{1}, \mathcal{T}_{2}\right)-\operatorname{sint}\left(f^{-1}(A), r, s\right)
$$

and

$$
f^{-1}(B)=\left(\mathcal{T}_{2}, \mathcal{T}_{1}\right)-\operatorname{sint}\left(f^{-1}(B), s, r\right) .
$$

Hence $f^{-1}(A)$ is an $\operatorname{IF}\left(\mathcal{T}_{1}, \mathcal{T}_{2}\right)-(r, s)$-semiopen set and $f^{-1}(B)$ is an IF $\left(\mathcal{T}_{2}, \mathcal{T}_{1}\right)-(s, r)$-semiopen set in $X$. Therefore $f$ is IF pairwise $(r, s)$-semicontinuous.

Theorem 3.8. Let $f:\left(X, \mathcal{T}_{1}, \mathcal{T}_{2}\right) \rightarrow\left(Y, \mathcal{U}_{1}, \mathcal{U}_{2}\right)$ be a bijective mapping from an ISBTS $X$ to an ISBTS $Y$ and $r, s \in I_{0}$. Then $f$ is IF pairwise $(r, s)$-semicontinuous if and only if

$$
\mathcal{U}_{1}-\operatorname{int}(f(A), r) \subseteq f\left(\left(\mathcal{T}_{1}, \mathcal{T}_{2}\right)-\operatorname{sint}(A, r, s)\right)
$$

and

$$
\mathcal{U}_{2}-\operatorname{int}(f(A), s) \subseteq f\left(\left(\mathcal{T}_{2}, \mathcal{T}_{1}\right)-\operatorname{sint}(A, s, r)\right)
$$

for each intuitionistic fuzzy set $A$ in $X$.

Proof. Let $A$ be an intuitionistic fuzzy set in $X$. Since $f$ is one-to-one, by Theorem 3.7, we have

$$
\begin{aligned}
f^{-1}\left(\mathcal{U}_{1}-\operatorname{int}(f(A), r)\right) & \subseteq\left(\mathcal{T}_{1}, \mathcal{T}_{2}\right)-\operatorname{sint}\left(f^{-1}(f(A)), r, s\right) \\
& =\left(\mathcal{T}_{1}, \mathcal{T}_{2}\right)-\operatorname{sint}(A, r, s)
\end{aligned}
$$

and

$$
\begin{aligned}
f^{-1}\left(\mathcal{U}_{2}-\operatorname{int}(f(A), s)\right) & \subseteq\left(\mathcal{T}_{2}, \mathcal{T}_{1}\right)-\operatorname{sint}\left(f^{-1}(f(A)), s, r\right) \\
& =\left(\mathcal{T}_{2}, \mathcal{T}_{1}\right)-\operatorname{sint}(A, s, r) .
\end{aligned}
$$

Because $f$ is onto, we obtain

$$
\begin{aligned}
\mathcal{U}_{1}-\operatorname{int}(f(A), r) & =f\left(f^{-1}\left(\mathcal{U}_{1}-\operatorname{int}(f(A), r)\right)\right) \\
& \subseteq f\left(\left(\mathcal{T}_{1}, \mathcal{T}_{2}\right)-\operatorname{sint}(A, r, s)\right)
\end{aligned}
$$

and

$$
\begin{aligned}
\mathcal{U}_{2}-\operatorname{int}(f(A), s) & =f\left(f^{-1}\left(\mathcal{U}_{2}-\operatorname{int}(f(A), s)\right)\right) \\
& \subseteq f\left(\left(\mathcal{T}_{2}, \mathcal{T}_{1}\right)-\operatorname{sint}(A, s, r)\right) .
\end{aligned}
$$

Conversely, let $B$ be an intuitionistic fuzzy set in $Y$. Since $f$ is onto, we obtain

$$
\begin{aligned}
\mathcal{U}_{1}-\operatorname{int}(B, r) & =\mathcal{U}_{1}-\operatorname{int}\left(f\left(f^{-1}(B)\right), r\right) \\
& \subseteq f\left(\left(\mathcal{T}_{1}, \mathcal{T}_{2}\right)-\operatorname{sint}\left(f^{-1}(B), r, s\right)\right)
\end{aligned}
$$

and

$$
\begin{aligned}
\mathcal{U}_{2}-\operatorname{int}(B, s) & =\mathcal{U}_{2}-\operatorname{int}\left(f\left(f^{-1}(B)\right), s\right) \\
& \subseteq f\left(\left(\mathcal{T}_{2}, \mathcal{T}_{1}\right)-\operatorname{sint}\left(f^{-1}(B), s, r\right)\right) .
\end{aligned}
$$

Because $f$ is one-to-one, we have

$$
\begin{aligned}
f^{-1}\left(\mathcal{U}_{1}-\operatorname{int}(B, r)\right) & \subseteq f^{-1}\left(f\left(\left(\mathcal{T}_{1}, \mathcal{T}_{2}\right)-\operatorname{sint}\left(f^{-1}(B), r, s\right)\right)\right) \\
& =\left(\mathcal{T}_{1}, \mathcal{T}_{2}\right)-\operatorname{sint}\left(f^{-1}(B), r, s\right)
\end{aligned}
$$

and

$$
\begin{aligned}
f^{-1}\left(\mathcal{U}_{2}-\operatorname{int}(B, s)\right) & \subseteq f^{-1}\left(f\left(\left(\mathcal{T}_{2}, \mathcal{T}_{1}\right)-\operatorname{sint}\left(f^{-1}(B), s, r\right)\right)\right) \\
& =\left(\mathcal{T}_{2}, \mathcal{T}_{1}\right)-\operatorname{sint}\left(f^{-1}(B), s, r\right) .
\end{aligned}
$$


Therefore by Theorem 3.7. $f$ is an intuitionistic fuzzy pairwise $(r, s)$-semicontinuous mapping.

\section{Conflict of Interest}

No potential conflict of interest relevant to this article was reported.

\section{Acknowledgments}

This work was supported by the research grant of Chungbuk National University in 2012.

\section{References}

[1] C. L. Chang, "Fuzzy topological spaces," Journal of Mathematical Analysis and Applications, vol. 24, no. 1, pp. 182190, Oct. 1968. http://dx.doi.org/10.1016/0022-247X(68) 90057-7

[2] A. P. Šostak, "On a fuzzy topological structure," Rendiconti Circolo Matematico Palermo (Supplement Series II), vol. 11, pp. 89-103, 1985.

[3] A. P. Šostak, "Two decades of fuzzy topology: basic ideas, notions, and results," Russian Mathematical Surveys, vol. 44, no. 6, pp. 125-186, 1989. http://dx.doi.org/10.1070/ RM1989v044n06ABEH002295

[4] A. A. Ramadan, "Smooth topological spaces," Fuzzy Sets and Systems, vol. 48, no. 3, pp. 371-375, Jun. 1992. http: //dx.doi.org/10.1016/0165-0114(92)90352-5

[5] D. Çoker, "An introduction to intuitionistic fuzzy topological spaces," Fuzzy Sets and Systems, vol. 88, pp. 81-89, 1997.

[6] D. Çoker and M. Demirci, "An introduction to intuitionistic fuzzy topological spaces in Sostak's sense," BUSEFAL, vol. 67, pp. 67-76, 1996

[7] K. T. Atanassov, "Intuitionistic fuzzy sets," Fuzzy Sets and Systems, vol. 20, no. 1, pp. 87-96, Aug. 1986. http: //dx.doi.org/10.1016/S0165-0114(86)80034-3
[8] T. K. Mondal and S. Samanta, "On intuitionistic gradation of openness," Fuzzy Sets and Systems, vol. 131, no. 3, pp. 323-336, Nov. 2002. http://dx.doi.org/10.1016/ S0165-0114(01)00235-4

[9] A. Kandil, "Biproximities and fuzzy bitopological spaces," Simon Stevin, vol. 63, no. 1, pp. 45-66, 1989.

[10] E. P. Lee, "Pairwise semicontinuous mappings in smooth bitopological spaces," Journal of Korean Institute of Intelligent Systems, vol. 12, no. 3, pp. 269-274, Jun. 2002.

[11] E. P. Lee, Y. B. Im, and H. Han, "Semiopen sets on smooth bitopological spaces," Far East Journal of Mathematical Sciences, vol. 3, pp. 493-511, 2001.

[12] P. K. Lim, S. R. Kim, and K. Hur, "Intuitionistic smooth topological spaces," Journal of Korean Institute of Intelligent Systems, vol. 20, no. 6, pp. 875-883, Oct. 2010. http://dx.doi.org/10.5391/JKIIS.2010.20.6.875

[13] E. P. Lee, "Semiopen sets on intuitionistic fuzzy topological spaces in Sostak's sense," Journal of Korean Institute of Intelligent Systems, vol. 14, no. 2, pp. 234-238, Apr. 2004. http://dx.doi.org/10.5391/JKIIS.2004.14.2.234

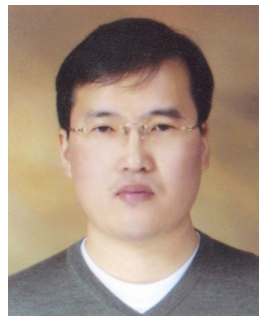

Jin Tae Kim received the $\mathrm{Ph}$. D. degree from Chungbuk National University in 2012. His research interests include general topology and fuzzy topology. He is a member of KIIS and KMS.

E-mail: kjtmath@hanmail.net

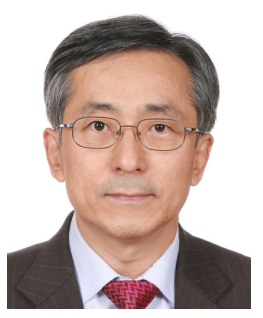

Seok Jong Lee received the M. S. and Ph. D. degrees from Yonsei University in 1986 and 1990, respectively. He is a professor at the Department of Mathematics, Chungbuk National University since 1989. He was a visiting scholar in Carleton University from 1995 to 1996, and Wayne State University from 2003 to 2004 . His research interests include general topology and fuzzy topology. He is a member of KIIS, KMS, and CMS.

E-mail: sjl@cbnu.ac.kr 